\title{
Reinforcement pattern learning: Do rats remember all prior events?
}

\author{
HISASHI YAZAWA and OSAMU FUJITA \\ University of Tsukuba, Niihari, Ibaraki, Japan
}

\begin{abstract}
Three experiments in a runway investigated how rats remember events occurring in temporal order. In Experiment 1, the reinforced trial was always the last (fifth) trial of the day for Group 4NR, the first trial for Group R4N, and the third trial for Group 2NR2N. For Group NIrg, one of Trials 2.5 was randomly reinforced. The running speeds for Group 2NR2N, an inverted V with its peak on Trial 3, were of special interest. An inverted V was also obtained for Group S-ITI (Experiment 2), which received the 2NR3N schedule at a 30-sec ITI, and for Group L-ITI (Experiment 3), which had a 30-min ITI. However, slow running on Trial 4 and not slow running on Trial 6 were demonstrated for Groups L-INT (Experiment 2) and S-INT (Experiment 3), which received the same schedule as Groups S-ITI and L-ITI, except for a long or short interval inserted between Trials 3 and 4 . These results suggest that rats remember all prior events occurring at an equal ITI whether the ITI is short or long, whereas they reset their memory when a longer or shorter interval elapses after the reinforced trial.
\end{abstract}

According to Capaldi's $(1967,1971)$ sequential hypothesis, the memory of nonreinforcement, $S^{N}$, is modified by successive nonreinforced trials and this memory regulates the partial reinforcement effect (PRE) through its behavioral control. Moreover, the sequential hypothesis has recently been extended to discrimination learning. It has become clear that partial reinforcement and conventional discrimination learning have much in common [e.g., brightness differential conditioning (Haggbloom, 1980, 1982), reinforcement pattern learning (Capaldi, 1979), and serial pattern learning (Capaldi \& Verry, 1981, Self \& Gaffan, 1983)].

Reinforcement pattern learning refers to discrimination learning based on cues provided by a fixed and repeated sequence of reinforced and nonreinforced trials, or a regular reinforcement schedule (see Capaldi, 1979). Reinforcement pattern learning has been examined employing a single alternation schedule (Campbell, Crumbaugh, Rhodus, \& Knouse, 1971) and a double alternation schedule (Bloom \& Capaldi, 1961). Capaldi (1979) investigated reinforcement pattern learning by employing two unique schedules, 4NR, four nonreinforced trials preceding a reinforcement trial each day, and $\mathrm{R} 4 \mathrm{~N}$, a reinforced trial followed by four nonreinforced trials each day. He reported that discriminative responding, that is, slower running on the nonreinforced trials than on the reinforced trial, occurred under both schedules, but that later in acquisition Group 4NR's responding was slow only on the initial nonreinforced trial. Capaldi explained these results in terms of the sequential hypothesis. Briefly, under the $4 \mathrm{NR}$ schedule, each of the memories occurring on the first

Requests for reprints should be sent to Hisashi Yazawa, Institute of Psychology, University of Tsukuba, Sakura-mura, Niihari-gun, Ibaraki 305 , Japan. four trials of the day, $S^{T} 1, S^{N} 1, S^{N} 2$, and $S^{N} 3$, respectively, was nonreinforced. $S^{\mathrm{T}} 1$ stands for the memory associated with the first trial of the day. $\mathrm{S}^{\mathrm{N}} 4$, the memory occurring on the last trial of the day, acquired associative strength, and this associative strength generalized strongly to the other trial memories, except for the highly dissimilar $S^{\mathrm{T}} 1$. On the other hand, under the R4N schedule, associative strength from the reinforced $S^{T} 1$ cue hardly generalized to the memory associated with each of the subsequent four trials of the day, or $S^{R}, S^{N} 1, S^{N}$, and $S^{N} 3$, respectively.

Capaldi's experiment provided a reinforced trial either in the first trial or the last trial of the day, with all other trials being nonreinforced. Here, if a reinforced trial is interjected in the middle of four daily nonreinforced trials, a very interesting reinforcement pattern results: two nonreinforced trials precede and follow a reinforced trial, or 2NR2N. Responding under the 2NR2N schedule is of interest because we might expect two alternative results, one following from the sequential hypothesis, as seen in Capaldi (1979), and the other following from his multiple hedonic memory hypothesis of serial pattern learning (Capaldi \& Verry, 1981). Although evidence for both hypotheses has been provided by Capaldi and others, there seems to be a substantial difference between these two hypotheses concerning specification of the memory available on the trial that followed the reinforced trial.

According to the sequential hypothesis, $\mathrm{S}^{\mathrm{N}}$ is modified by successive nonreinforced trials, and this stimulus modification continues until a reinforced trial occurs (Capaldi, 1967). This means that rats reset the $\mathrm{N}$-length cue on a reinforced trial and then stimulus modification of the $\mathrm{N}$-length cue begins anew on the next nonreinforced trial. Thus, under the $2 \mathrm{NR} 2 \mathrm{~N}$ schedule, memory on each trial can be traced as follows: On Trial $1, S^{T} 1$ is produced; 
on Trial 2 , the memory associated with $\mathrm{N}$-length 1 , or $S^{N} 1$, is produced; on Trial 3, it is $S^{N} 2$; and on Trial 4, $\mathrm{S}^{\mathbf{R}}$. It should be noted that on Trial 5 the memory associated with $\mathrm{N}$-length 1 , or $\mathrm{S}^{\mathrm{N}} 1$, is produced again, as on Trial 2 . Under this schedule, $S^{N} 2$ on Trial 3 is reinforced and each of the other memories is nonreinforced. It is also assumed that the reinforced cue, $S^{N_{2}} 2$, is more similar to $S^{\mathrm{N}} 1$ than to $S^{\mathrm{R}}$, and $\mathrm{S}^{\mathrm{T}} 1$ on Trial 1 is substantially different from $S^{N} 2$. Thus, it is predicted that the relative running speed on each trial is Trial $3>$ Trial 2 $=$ Trial $5>$ Trial $4>$ Trial 1 .

On the other hand, in serial pattern learning experiments, Capaldi and Verry (1981) proposed that rats could use multiple memory $S 1+S^{N} 1$ in a 1-0-20 series and $S 20+S^{N} 1$ in a 20-0-0 series on Trial 3 , and so rats could predict whether Trial 3 was 20 or 0 . S1 and S20 stand for memory associated with 1 pellet and 20 pellets, respectively. The hypothesis that rats could use multiple memory was also confirmed by Capaldi, Verry, and Nawrocki (1982). In contrast to the sequential hypothesis, however, the multiple hedonic memory hypothesis suggested that stimulus modification of $\mathrm{S}^{\mathrm{N}}$ did not begin after a reinforced trial, but that the memory of reinforcement $S^{R}$ was combined with the preceding N-length cue. So it is assumed that multiple memory $S^{N} 2+S^{R}$ is produced on Trial 4. In other words, all prior events are remembered, not just the reinforced trial. $S^{N} 2+S^{R}+S^{N} 1$ is assumed to be produced on Trial 5. Thus, after reinforced Trial 3, the discrepancy between the memory produced on each trial and the reinforced memory $S^{N} 2$ should increase. Therefore, running speed should increase over the first three trials of the day, reach its highest level on Trial 3, and then should decline over the last two trials. In other words, it should show an inverted $V$ curve with a peak on Trial 3.

Experiment 1 was designed to test whether rats reset the $\mathrm{N}$-length cue on a reinforced trial or used multiple hedonic memory of all prior events despite the intervention of the reinforced trial; we used 4NR and R4N schedules along with a 2 NR2N schedule. In addition, reinforcement pattern learning has been examined only in regular reinforcement schedules, under which the position of the reinforced trial is fixed across days. In order to examine whether rats demonstrate discriminative responding when the position of the reinforced trial is not fixed, we designed a unique schedule, called NIrg, under which the first trial of the day was always nonreinforced and one of the remaining four trials was irregularly reinforced.

\section{EXPERIMENT 1}

\section{Method}

\section{Subjects}

The subjects were 52 naive male albino rats of the WistarImamichi strain obtained from the colony at the University of Tsukuba. They were about 100 days old at the beginning of the experiment.

\section{Apparatus}

The gray straight-alley runway, which was covered with Plexiglas, was $147 \mathrm{~cm}$ long and $12 \mathrm{~cm}$ wide and had 16-cm-high sides. It had a 23-cm-long startbox, a 93-cm-long runway, and a 31-cmlong goalbox. Startbox and goalbox were separated from a runway by guillotine doors. A timing circuit (Kokusai Electronics Corp., Japan) recorded running times to the nearest .1 sec. When the first guillotine door was raised, the first clock started. Interrupting a photobeam $16 \mathrm{~cm}$ from a startbox stopped Clock 1 (start time) and started Clock 2. Interrupting a beam $70 \mathrm{~cm}$ from the first beam stopped Clock 2 (run time) and started Clock 3. Clock 3 was stopped (goal time) by the interruption of a beam $28 \mathrm{~cm}$ from the second beam and $10 \mathrm{~cm}$ from the endwall of the goalbox, where the metal food cup, $3.0 \mathrm{~cm}$ in diameter and $0.7 \mathrm{~cm}$ high inside, was attached.

\section{Procedure}

Preliminary training. A $12-\mathrm{g}$ food-deprivation schedule began on Day 1 of preliminary training. Water was always available. By Day 12 , each rat reached $85 \%-90 \%$ of its ad-lib weight, and this weight was maintained for the rest of the experiment. On Days 912, all rats were handled daily for $3 \mathrm{~min}$. On Days 11-12, after handling, groups of four rats were allowed to explore the apparatus for $8 \mathrm{~min}$ a day. In addition, on Days 5-12, each rat was given $1545-\mathrm{mg}$ food pellets in the neutral cage and then returned to the home cage.

Experimental training. Following preliminary training, experimental training, which consisted of five trials per day for 24 days, began. On Day 1, the subjects were divided into four groups of 13 rats each. In the experimental phase, all four groups-Group 4NR, Group R4N, Group 2NR2N, and Group NIrg-were reinforced on only one trial each day and nonreinforced on all remaining trials. As in Capaldi (1979), for Group 4NR the reinforced trial was always the last (fifth) trial of the day, and for Group R4N it was always the first trial of the day. For Group 2NR2N, the third trial of the day was always reinforced. For Group NIrg, the first trial was always nonreinforced and one of the remaining four trials was randomly reinforced. The reinforced trial for Group NIrg was the second trial on Days 2,6,10,14,18, and 22, the third trial on Days 3, 7, 11, 15, 19, and 23, the fourth trial on Days 1, 5, 9, 13,17 , and 21 , and the fifth trial on Days $4,8,12,16,20$, and 24 .

The rats were given $1545-\mathrm{mg}$ food pellets on reinforced trials and removed from the goalbox immediately after eating the last pellet. On nonreinforced trials, they were confined to the goalbox for $10 \mathrm{sec}$. The rats were run in squads of four, one from each of the four groups. The rats were taken in squads from the breeding room to the experimental room and were put into the detention cage individually. The start door was raised $3 \mathrm{sec}$ after a rat had been placed in the start box. Each rat received its first trial before any rat received its second trial, and so on. Daily running order of rats within a squad was randomized. The intertrial interval was approximately 15 to $25 \mathrm{~min}$ in early experimental training and 5 to $8 \mathrm{~min}$ in later training. If a rat failed to traverse any runway section within $60 \mathrm{sec}$, it was placed directly into the goalbox and assigned a time score of $60 \mathrm{sec}$ in each of the remaining sections. About $10 \mathrm{~min}$ after the last rat in the squad finished the last trial, the rats were returned to the breeding room and given $12 \mathrm{~g}$ of food pellets each.

\section{Results}

All time measures were converted into speeds $(1 / \mathrm{sec})$. Since similar results were obtained in each alley section, only the results for total time are reported here. Figure 1 shows the total speeds for each of four groups on each of the five trials of the 4-day blocks in experimental training. 


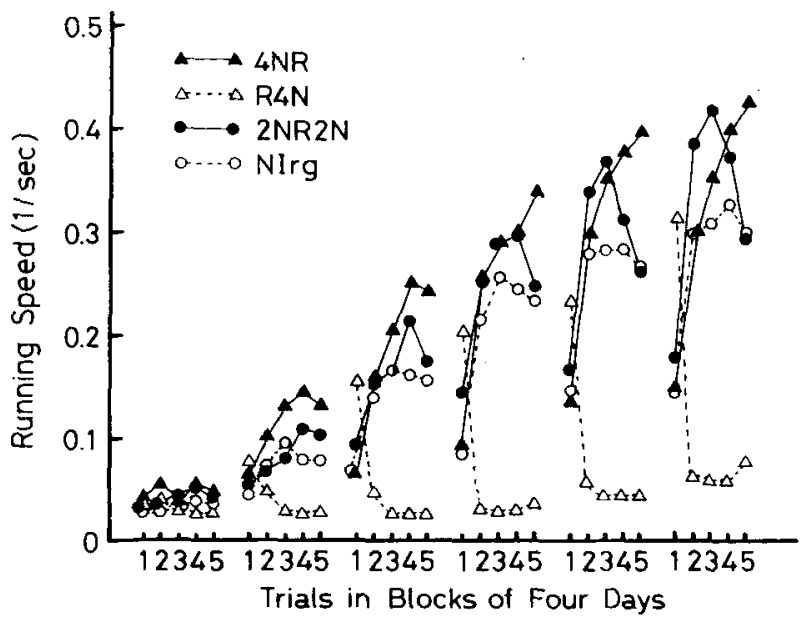

Figure 1. Total running speeds (1/sec) of four groups on each of the five trials of 4-day blocks in training. Experiment 1.

Figure 1 shows that each group developed pattern running with increased training. In the last block, for Groups $4 N R$, R4N, and 2NR2N, the trial on which the peak of the running speed occurred was the reinforced trial. Within analyses of variance of trials (5) in the last block for each group indicated that differences among trials were significant for Group 4NR $[F(4,48)=23.17, p<.01]$, Group R4N $[F(4,48)=22.20, p<.01]$, Group 2NR2N $[F(4,48)=18.75, p<.01]$, and Group NIrg $[F(4,48)$ $=22.19, \mathrm{p}<.01]$. The running curve for each group was examined separately in the last block. In order to analyze significant differences among trials, Tukey's posttest was conducted using the appropriate error term from a within analysis of variance for each group.

For Group 4NR, running speed increased progressively over the five trials of the day and reached its highest level on Trial 5, the reinforced trial. Tukey's posttest indicated that the speeds on Trials 4 and 5 significantly differed from those on Trials 1 and 2 (ps $<.01$ ). Trials 2 and 3 differed from Trial 1 (ps < .01). However, differences between Trials 3, 4, and 5 did not reach an acceptable level of significance.

For Group R4N, running was faster on reinforced Trial 1 than on every nonreinforced trial. Tukey's posttest indicated that Trial 1 differed from every other trial (ps $<.01$ ), with no other differences being significant.

For Group 2NR2N, running speed increased progressively over the first three trials of the day and peak speed occurred on Trial 3. Then speed declined gradually over the last two trials. Tukey's posttest indicated that Trial 1 differed from every other trial (ps $<.01$ ) and that the rats ran more slowly on Trial 5 than on Trials 2,3 , and 4 (ps $<.05$ or better). The differences between Trials 2,3 , and 4 did not reach an acceptable level of significance.

For Group NIrg, the rats ran more slowly on Trial 1, which was always nonreinforced, than on every other trial, each of which was partially (one-quarter) reinforced.
Tukey's posttest indicated that Trial 1 differed from every other trial ( $p s<.01$ ), with no other differences being significant. In order to examine the possibility that running pattern depended on the position of the reinforced trial, Group NIrg's running speeds on each of 4 days in the last block were examined. It was found that the running pattern on any one day was much the same as that on another. Tukey's posttest indicated speed was slower on Trial 1 than on every other trial (ps $<.01$ ); no other differences on any day were significant.

In addition, a between analysis of variance of group (4) $\times$ trial (5) in the last block indicated that the group $\times$ trial interaction was significant $[F(12,192)=24.57$, $p<.01]$. A subsequent Tukey's posttest was conducted to compare the speeds of each group on the reinforced trial. It was shown that Group 4NR's speeds on Trial 5 and Group 2NR2N's speeds on Trial 3 were faster than Group NIrg's on Trials 2, 3, 4, and 5 and Group R4N's on Trial 1 ( $\mathrm{p}<.05$ or better $)$.

\section{Discussion}

In the context of partial reinforcement, the sequential hypothesis (Capaldi, 1967, 1971) assumes that the Nlength cue is reset and conditioned to the instrumental response on the reinforced trial. Then $S^{R}$ is assumed to occur on the next trial. If this notion is extended to reinforcement pattern learning, it is assumed that rats use the $\mathrm{N}$-length cue and $\mathrm{S}^{\mathbf{R}}$ separately as discriminative stimuli. For example, under a single-alternation schedule, $S^{\mathbf{R}}$ always occurs on nonreinforced trials and so does the $\mathrm{S}-$ cue, whereas $S^{N}$ always occurs on reinforced trials, as does the $S+$ cue (see Capaldi, 1979).

However, the inverted-V running pattern obtained under the 2 NR2N is not consistent with the notion suggested by the sequential hypothesis. According to the sequential hypothesis, the N-length 1 cue $\left(S^{N} 1\right)$ occurs on both Trial 2 and Trial 5, so it cannot explain the result that the running speeds were Trial $2>$ Trial 5 . With regard to the speeds on Trials 4 and 5 , since the sequential hypothesis assumes that the reinforced $S^{N_{2}}$ on Trial 3 is more similar to $S^{\mathrm{N}} 1$ on Trial 5 than to $S^{\mathrm{R}}$ on Trial 4 , it is predicted that the running speed would be Trial $5>$ Trial 4. But the result obtained showed Trial $4>$ Trial 5 .

In contrast, the $2 \mathrm{NR} 2 \mathrm{~N}$ result is in accordance with the notion suggested by the multiple hedonic memory hypothesis (Capaldi \& Verry, 1981; Capaldi et al., 1982). According to the multiple hedonic memory view, because the $\mathrm{N}$-length cue is not reset on reinforced Trial 3, memory on each trial is as follows: On Trial $1, S^{T} 1$ is produced; on Trial 2, it is $\mathrm{S}^{\mathrm{N}} 1$; on Trial $3, \mathrm{~S}^{\mathrm{N}} 2$; on Trial $4, S^{N_{2}}+S^{R}$; on Trial $5, S^{N} 2+S^{R}+S^{N} 1$. Since the resemblance between the memory on each trial and the reinforcement cue $S^{N} 2$ increases until Trial 3 and then decreases following Trial 3, running speed is predicted to be an inverted $\mathrm{V}$, with its peak on Trial 3 , just the relationship found in this experiment. The results for Group 
2NR2N suggest that rats used multiple hedonic memory of all prior hedonic events in the series despite the intervention of the reinforced trial.

As with the 2NR2N schedule, each running pattern obtained under the other three schedules shows that rats use multiple hedonic memory of all prior events. Under the 4NR schedule, the memories occurring on Trials 1-5 are of $S^{\mathrm{T}} 1, S^{\mathrm{N}} 1, S^{\mathrm{N}} 2, S^{\mathrm{N}} 3$, and $S^{\mathrm{N}} 4$, respectively. As the trials progress, the similarity between the memory which occurred on each of Trials 1-4 and the reinforced memory $\mathrm{S}^{\mathrm{N}} 4$ on Trial 5 increases. Thus, running speed increased with the increasing number of trials, as shown in this experiment. Under the R4N schedule, the memory which occurred on each of Trials $1-5$ is $S^{T} 1, S^{R}, S^{R}+S^{N} 1$, $S^{R}+S^{N} 2, S^{R}+S^{N} 3$, respectively. Under the NIrg schedule, $S^{\mathrm{T}} 1$ on Trial 1 was always nonreinforced, but $S^{N_{1}} 1$ on Trial $2, S^{N_{2}}$ on Trial $3, S^{N_{3}} 3$ on Trial $4, S^{N^{2}} 4$ on Trial 5 were each $25 \%$ partially reinforced. According to Capaldi and Morris (1974), the habit strength acquired by $S^{\mathrm{T}} 1$ does not generalize very strongly to the other memories, and vice versa, because $S^{T} 1$ does not have the same stimulus dimension as $\mathbf{S}^{\mathbf{R}}$ or $\mathbf{S}^{\mathbf{N}}$. That is why, under the R4N schedule, rats ran fast only on reinforced Trial 1, whereas, under the NIrg schedule, they ran slowly only on nonreinforced Trial 1 . In the same way, the dimensional difference between $S^{T} 1$ and $S^{N}$ can explain the result that the speed was slower on reinforced Trial 1 under the R4N schedule than it was on Trial 3 under the 2NR2N schedule or on Trial 5 under the 4NR schedule. Each of Group NIrg's running speeds on Trials 2-5 were slower than Group 4NR's speed on Trial 5 and Group 2NR2N's speed on Trial 3. Since, in each case, the possible reinforced memory was only $25 \%$ partially reinforced for Group NIrg, the habit strength acquired would be weaker than that acquired under the $4 \mathrm{NR}$ and the 2NR2N schedules.

The result for Group R4N was consistent with Capaldi's (1979). However, Group 4NR in the present experiment demonstrated better patterning than did Capaldi's Group 4NR, which ran slowly only on Trial 1 . Capaldi employed a 10-sec delay of reinforcement and an ITI of about 10 min, whereas we employed immediate reinforcement and a 5-8-min ITI. According to Capaldi (1967), the asymptote of habit growth is lower under delayed than under immediate reinforcement. It is assumed that Group 4NR would fail to reflect pattern learning adequately because of the lower asymptote under the delayed reinforcement employed in Capaldi's experiment. The latter half of the present report will make it clear that the difference between a 10-min ITI and a 5-8-min ITI would not cause the discrepancy between our 4NR result and Capaldi's.

Recently, Self and Gaffan (1983) suggested that animals might be able to remember whether or not a particular hedonic event had occurred previously during the trial. Applied to the present study, this would mean that the rats would learn to run fast until the reinforced trial and to run slowly following that trial. However, it cannot explain the running pattern under the NIrg schedule. If rats used that strategy, running speed should have declined after the reinforced trial. However, Group NIrg's running pattern did not depend on reinforcement position.

\section{EXPERIMENT 2}

An inverted-V running speed under the 2 NR2N schedule in Experiment 1 showed that rats could use the multiple hedonic memory of all prior hedonic events despite the intervention of a reinforced trial. Do rats remember all given hedonic events in any situation? Regarding this question, Capaldi and Verry (1981) and Capaldi et al. (1982) suggest that rats group, or "chunk," hedonic events occurring with a short interval and treat them as being separate from hedonic events that may occur subsequently after a long interval. This means that rats would reset the multiple memory of the prior events if a long interval was inserted after the reinforced trial.

In Experiment 2, the 2NR3N (NNRNNN) schedule (one nonreinforced trial added to the 2NR2N schedule) was employed with each of two groups. The two groups differed only in the length of the interval inserted between Trials 3 and 4. One group, named S-ITI (short ITI), received NNRNNN at each 30-sec ITI. This group should confirm the 2NR2N result obtained in Experiment 1. The other group, named L-INT (long interval), received NNR with a 30-sec ITI followed by a 30-min interval, and then an NNN with a 30-sec ITI. There are two alternative possibilities regarding the running patterns for these two groups. If rats in Group L-INT do not use the cue related to the long interval, the running patterns for Groups LINT and S-ITI should be identical. On the other hand, if the rats reset the multiple memory of prior events at the long interval after the reinforced trial, these two groups should demonstrate different running patterns.

\section{Method}

\section{Subjects and Apparatus}

The subjects were 24 rats, as described in Experiment 1, and the apparatus was the same.

\section{Procedure}

Preliminary training was the same as in Experiment 1. On Day 13, the first day of experimental training, the rats were divided into two groups, Group S-ITI and Group L-INT, of 12 rats each. Experimental training consisted of six trials per day for 24 days. The rats were tested in squads of eight, four from each group. The running order within a squad was randomized daily. Group S-ITI was reinforced on Trial 3 and nonreinforced on all remaining trials, with a 30-sec ITI in each day. Group L-INT received the same reinforcement sequence except for the interval between Trial 3 and Trial 4, which was 30 min spent in the detention cage. During this interval, other rats in the squads were tested. Except for the differences noted above, all other procedures were identical to those employed in Experiment 1.

\section{Results}

Figure 2 shows, in blocks of 4 days, total speeds on each of the six trials for the two groups. Figure 2 shows that each group developed a different running pattern with 


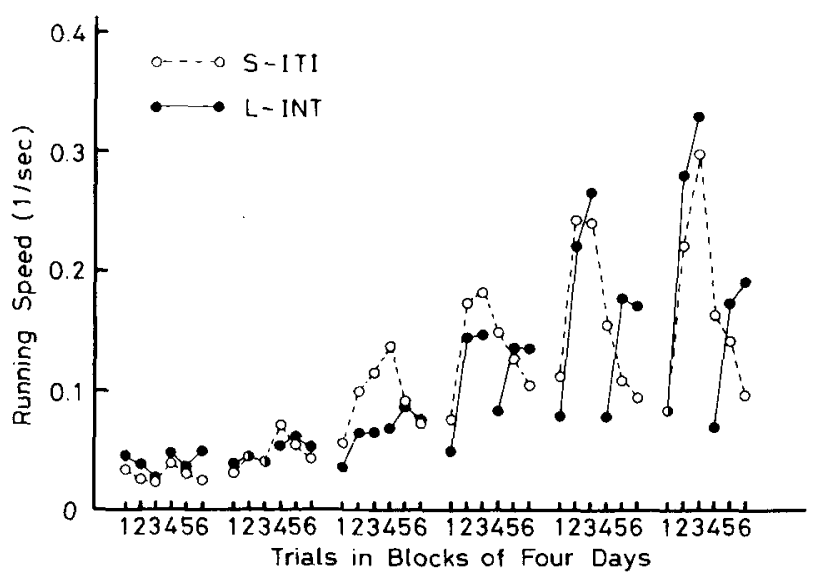

Figure 2. Total running speeds (1/sec) of Group S-ITI and Group L-INT on each of the six trials of 4-day blocks in training. Experiment 2.

increased training. Analysis of variance on speed, groups (2) $\times$ trials (6), indicated that the group $\times$ trial interaction first became significant on Block $3[\mathrm{~F}(5,110)=$ $3.45, \mathrm{p}<.01]$ and remained significant until the last block $[F(5,110)=4.96, p<.01]$. There was no significant main effect for groups. The main effect for trials was significant on Block $1[F(5,110)=3.38, p<.01]$ and remained significant until the last block $[F(5,110)=$ $37.35, \mathrm{p}<.011$.

In the last block, the running speed for Group S-ITI increased over the first three trials and reached its highest level on Trial 3, which was reinforced, then gradually declined over the last three trials. That is, Group S-ITI showed an inverted $\mathrm{V}$ running pattern with its peak on Trial 3. The within analysis of variance of trials (6) in the last block indicated that differences among trials were significant $[\mathrm{F}(5,55)=15.19, \mathrm{p}<.01]$. Tukey's posttest indicated that Trial 3 was faster than every other trial (ps $<.01$ ) and that both Trial 2 and Trial 4 differed from every other trial ( $\mathrm{p}<.05$ or better).

For Group L-INT, in the last block, the running speed increased over the first three trials and reached its highest level on Trial 3, as it did for Group S-ITI. However, it should be noted that, following Trial 3, Group L-INT demonstrated a quite different running pattern from that for Group S-ITI. Group L-INT ran very slowly on Trial 4, which followed the 30 -min interval. Then speed increased again on the last two trials. The within analysis of variance indicated that differences among trials were significant $[F(5,55)=27.38, p<.01]$ in the last block. Tukey's posttest indicated that Trial 3 was faster than every other trial (ps $<.05$ or better) except Trial 2 , and that both Trial 5 and Trial 6 differed from every other trial $(p<.05$ or better). Trial 4 did not differ from Trial 1 .

In addition, in the between-group comparison in the last block, Tukey's posttest indicated that Group L-INT differed from Group S-ITI both on Trial 4 and Trial 6 (ps $<.05$ ); no other differences were significant. There- fore, two running curves were identical until Trial 3, but crossed after that trial.

\section{Discussion}

Employing the 2NR3N schedule, an inverted-V running pattern was obtained for Group S-ITI, which replicated the 2NR2N result of Experiment 1. Moreover, Group S-ITI showed significant differences between Trials 2,3 , and 4, which did not reach an acceptance level of significance under the $2 \mathrm{NR} 2 \mathrm{~N}$. The result for Group S-ITI confirmed the notion that rats use the multiple hedonic memory of all prior events despite the intervention of the reinforced trial.

Group L-INT received the same 2 NR3N schedule as Group S-ITI, except for the interval between Trials 3 and 4. However, following Trial 3, Group L-INT demonstrated a quite different running pattern from that for Group S-ITI. The result for Group L-INT can be explained as follows. Group L-INT used the same memory as Group S-ITI did on each of Trials 1-3, that is, $S^{\mathrm{T}} 1$ on Trial $1, \mathrm{~S}^{\mathrm{N}} 1$ on Trial $2, \mathrm{~S}^{\mathrm{N}} 2$ on Trial 3. However, Group L-INT reset the multiple memory of prior events at the long interval after the reinforced Trial 3. Group L-INT used the nonhedonic memory on Trial $4, S^{N} 1$ or $S^{N} 2$ again on Trial 5 or Trial 6 . As the nonhedonic memory on Trial 4 was more dissimilar to the reinforced memory $S^{N_{2}}$ on Trial 3 than to $S^{N} 1$ on Trial 5 or $S^{N} 2$ on Trial 6 , running speed for Group L-INT declined substantially on Trial 4 and increased again on the last two trials. But running speed on Trial 6 was not faster than that on the reinforced Trial 3. It is possible that the rats used a 30-min related cue with $S^{N} 2$. In fact, the rats' ability to mark the passage of time is highly accurate. Mellgren, Mays, and Haddad (1983) showed that rats can discriminate 6 min from $12 \mathrm{~min}$ in the runway situation. However, if rats used only the 30-min related cue, running speed should not increase again on the last two trials. Even if a 30-min related cue was compounded with the multiple hedonic memory, the result for Group L-INT indicated that the rats reset the multiple hedonic memory at the long interval after the reinforced trial.

\section{EXPERIMENT 3}

In Experiment 2, it was found that rats used their multiple hedonic memory of all prior events presented with a short interval, and then reset it at the long interval inserted after the reinforced trial. These results coincide with Capaldi and Verry's (1981) notion that rats group hedonic events occurring at a short interval and treat them as being separate from those after a long interval. According to their concept, the results in Experiment 2 can be interpreted as showing that Group S-ITI grouped one long NNRNNN series, whereas Group L-INT grouped two short NNR and NNN series separately. Generally, a short interval is considered to be a 20-30-sec ITI, and a long interval, a 20-30-min ITI (e.g., Capaldi \& Verry, 1981; 
Capaldi et al., 1982; Experiment 2 in the present study). Do rats use multiple hedonic memory if all events are given at a long 30-min interval? To answer this question, we used a long-ITI group, Group L-ITI, which received NNRNNN at a constant 30-min ITI. If rats use the multiple hedonic memory even at a long interval, Group LITI should demonstrate an inverted-V running pattern, as Group S-ITI did in Experiment 2.

In Experiment 2, Group L-INT, which received NNRNNN at a short ITI, except for the long interval between Trial 3 and Trial 4, indicated that rats reset, at the long interval, their multiple hedonic memory of prior events. Do rats reset their memory only when a long interval is inserted after the reinforced trial when all other ITIs are short? Conversely, do rats reset their memory when a short interval is inserted immediately after the reinforced trial when all other ITIs are long? In order to answer this question, we used a short-interval group, Group S-INT, which was trained as was Group L-ITI except that a 30-sec interval elapsed between Trials 3 and 4 .

\section{Method}

\section{Subjects and Apparatus}

The subjects were 24 rats, as described in Experiments 1 and 2 . The apparatus was the same as that in the previous experiments.

\section{Procedure}

Preliminary training was the same as in the previous experiments. On Day 13, the rats were divided into two groups, Group L-ITI and Group S-INT, of 12 rats each. Group L-ITI was reinforced on Trial 3 and nonreinforced on all other trials, with a 30-min ITI. Group S-INT received the same reinforcement sequence except for the interval between Trial 3 and Trial 4. Group S-INT spent $30 \mathrm{sec}$ in the detention cage in this interval. The rats were tested in squads of eight, four from each of the two groups. Except for the treatment between Trial 3 and Trial 4 for Group S-INT, no rat received the next trial before all the other rats had received the previous one. To allow for a 30-min ITI for each rat, the interval between each rat's run was 1 to $10 \mathrm{~min}$.

\section{Results}

Figure 3 shows that, with increased training, each group developed a characteristic running pattern. Analysis of variance indicated that the group $\times$ trial interaction effect first became significant on Block $2[F(5,110)=4.49$, $\mathrm{p}<.01]$ and remained significant until the last block $[F(5,110)=7.26, p<.01]$. There was no main effect for groups. The main effect for trials was significant on Block $1[\mathrm{~F}(5,110)=15.67, \mathrm{p}<.01]$ and remained significant until the last block $[F(5,110)=50.97, p<.01]$. The major finding in Experiment 3 can be seen in the last block and summarized as follows. Group L-ITI showed an inverted-V running pattern with its peak on Trial 3. Group S-INT showed the same running pattern as Group L-ITI did until Trial 3. However, Group S-INT ran very slowly on Trial 4, which followed the 30-sec interval, and then fast again on the last two trials.

A statistical analysis on running speed in the last block confirmed the results mentioned above. The within anal-

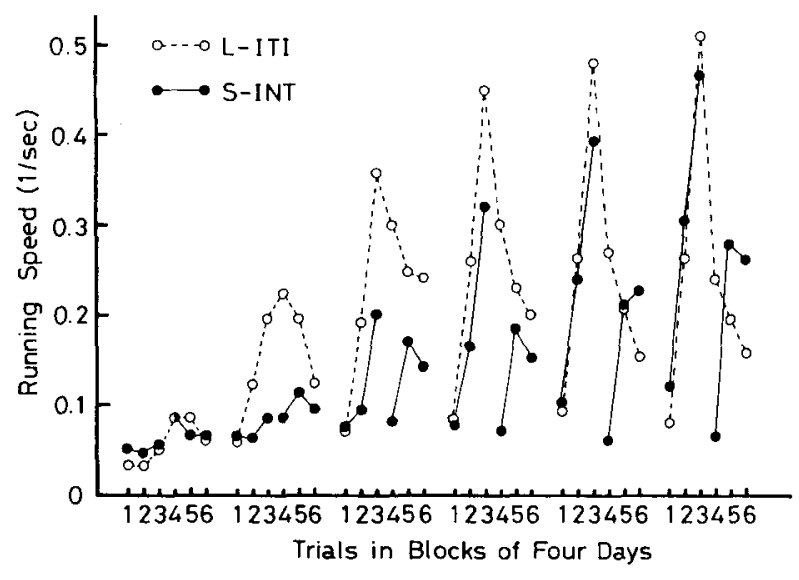

Figure 3. Total running speeds (1/sec) of Group L-ITI and Group S-INT on each of the six trials of 4-day blocks in training. Experiment 3.

ysis of variance for Group L-ITI indicated that differences among trials were significant $[F(5,55)=25.52$, $\mathrm{p}<.01$ ]. Tukey's posttest indicated that Trial 3 was faster than every other trial (ps $<.01$ ) and that Trials 2 and 4 differed from every other trial $(p<.05$ or better) except Trial 5. For Group S-INT, differences among trials were significant $[F(5,55)=34.49, p<.01]$. Tukey's posttest indicated that Trial 3 was faster than every other trial (ps $<.01)$ and that Trials 2,5 , and 6 were each faster than Trial 1 or Trial 4 (ps $<.01$ ). In addition, in the between-group comparison, Tukey's posttest indicated that Group S-INT differed from Group L-ITI in Trials 4 and 6 (ps $<.05$ ). The difference in Trial 5 did not reach an acceptable level. In other words, two running curves were identical until Trial 3 but crossed after that trial.

\section{GENERAL DISCUSSION}

The multiple hedonic memory hypothesis originally came from the serial pattern learning experiment of Capaldi and Verry (1981), in which concurrent pairs of sequence, such as 1-0-20 and 20-0-0 or $0-20-20$ and 20$0-0$, were employed. Capaldi and Verry, however, wondered if rats would use multiple hedonic memory without the use of such pairs of sequence. Self and Gaffan (1983) also thought that the multiple memory solution was required in the concurrent sequence paradigm, but supposed that rats would use the memory of just one preceding hedonic event in the single sequence paradigm.

However, an inverted V obtained for Group 2NR2N (Experiment 1), Group S-ITI (Experiment 2), and Group L-ITI (Experiment 3) confirms that rats use the multiple hedonic memory of all prior events, even in a single sequence, which is less complex than a concurrent one. The result for Group S-ITI is consistent with Capaldi and Verry (1981), who report the ability of rats to remember many events occurring at a 20-30-sec ITI. In addition to their study, all other demonstrations of multiple memory 
have been done at a short ITI (e.g., Capaldi et al., 1982; Self \& Gaffan, 1983). With regard to a long ITI, Jobe, Mellgren, Feinberg, Littlejohn, and Rigby (1977) suggested that, by earning a single-alternation patterning at a 24-h ITI, rats could remember one previous hedonic event occurring $24 \mathrm{~h}$ before. In other words, their study demonstrates memory for only a single hedonic event at a very long ITI. Therefore, the finding that Group L-ITI demonstrated the same running pattern as did Group SITI regardless of the difference in the ITI is of significance, because Group L-ITI in Experiment 3 is the first demonstration of multiple memory at a long, 30-min ITI. Since Group L-ITI received Trial 62 1/2 h after receiving Trial 1 , it is clear that rats can remember all six events over a time span of $2 \frac{1 / 2}{h}$.

By examining his results together with a variety of previous extinction data, Capaldi (1979) suggested that $S^{N}$ and $S^{R}$ were functional at all intervals. The inverted $\mathrm{V}$ obtained at both a long ITI and a short ITI demonstrates that not only $S^{\mathrm{N}}$ and $\mathrm{S}^{\mathrm{R}}$ singly but also multiple memory can be maintained over a relatively long period of time and is unaffected by delay. This feature of multiple memory is in accord with that of working memory proposed by Honig (1978), who classified a single-alternation pattern of reinforcement in a runway as a working memory task. Every schedule used in the present study can also be classified as a working memory task if Honig's statement regarding the initial stimulus is extended to the outcome of all prior trials, that is, memory of all prior events. In a series of radial maze experiments, $\mathrm{Ol}-$ ton and others have demonstrated that the ability of rats to remember the stimulus events occurring in spatial order is highly developed and accurate, and they conclude that the rat maintains a working memory of the arms it has entered throughout the trial (e.g., Olton, 1978; Olton \& Samuelson, 1976). Although Olton and others used a spatial maze task whereas the present experiments used a temporal sequence task, both experiments demonstrate that rats can remember all prior events or multiple events in the situation in which the events to be remembered change from trial to trial.

Concerning the role of the intertrial interval, a noteworthy finding is obtained in the present experiments. Groups S-INT (Experiment 3) demonstrated the same running pattern as Group L-INT (Experiment 2); this pattern differed from the inverted V obtained for Group SITI (Experiment 2) and Group L-ITI (Experiment 3). The extremely slow running on Trial 4 for both Group L-INT and Group S-INT cannot be explained by any absolute effects resulting from the passage of time between Trial 3 and Trial 4, because running on Trial 4 was not slow for Group S-ITI or Group L-ITI, which received the same procedure as Groups S-INT and L-INT between Trial 3 and Trial 4. Mellgren et al. (1983) suggest that a temporal stimulus serves as a discriminative cue when the length of the ITI predicts the trial outcome. Mellgren's notion may explain the slow running on nonreinforced Trial 4, which always followed a long interval for Group
L-INT or a short interval for Group S-INT. However, it cannot explain the differences among Trials $2,3,5$, and 6, each of which followed the same length of ITI.

Capaldi and Verry (1981) noted that " as long as trials occurred at a 25-30-sec interval, the rat instructed itself to remember all prior hedonic events in the series, no matter how far removed in time. However, once a relatively long interval had elapsed without a trial, it was no longer useful to remember prior hedonic events, and the rat used this cue as the instruction to forget all prior hedonic events" (p. 449). Capaldi and Verry made note of memory resetting only in a long interval; they did not make clear whether or not rats could remember prior events in a long interval. Considering the ability of rats to remember events given at a long ITI, as demonstrated for Group L-ITI, the running pattern for Group L-INT indicates that rats reset the memory of prior events at a long interval despite the fact that they can remember them after a long interval. Moreover, memory resetting operates even in a short interval inserted after the reinforced trial, as demonstrated for Group S-INT.

The similarity between multiple memory and working memory has already been discussed in this report. They are also similar in having a resetting mechanism that is independent of absolute time length. Olton (1978) indicates that, in the radial maze experiment, rats can reset working memory during a 1-min interval between tests. In contrast, it has been found that rats can remember all prior choices when a delay of $15 \mathrm{sec}$ to $2 \mathrm{~min}$ (Maki, Brokofsky, \& Berg, 1979) or delay of $4 \mathrm{~h}$ (Beatty \& Shavalia, 1980) is imposed between the 4th and 5th choices. These radial maze results show that whether all choices in a test session are finished or not is a determinant for memory resetting. The results of the present experiments suggest that memory resetting depends upon a relative time length which is compared with other intervals used in the schedule, that is, it depends upon the change in the ITI within a day. Therefore, from the results in Experiments 2 and 3 as follows, we conclude that rats remember all prior events occurring with an equal ITI, regardless of whether the ITI is long or short, but reset the memory of prior events when a shorter or a longer interval elapsed after the reinforced trial.

Group 2NR2N in Experiment 1 showed an inverted V, but it did not show statistical differences between Trial 3 and Trial 2 or between Trial 3 and Trial 4, which Groups S-ITI and L-ITI did. In Experiment 1, the ITI was 15 to $25 \mathrm{~min}$ in early training and 5 to $8 \mathrm{~min}$ in later training, since rats were run in rotation in squads of four. Capaldi and Minkoff (1967) and Ishida (1981) failed to find singlealternation patterning using a variable ITI. Whether the length of ITI is equal or not plays an important role in pattern discrimination, as shown in the present Experiments 2 and 3. It is possible that a variable ITI in Experiment 1 was responsible for the few significant differences between trials for Group 2NR2N.

The present experiments have shown that the use of equal intervals between trials is an important determiner 
of pattern learning. Until now, investigations have studied serial pattern learning in rats by using a short intertrial interval and a long interseries interval. Changing the lengths of intertrial or interseries intervals with the same serial pattern should produce the quite different performance. Such an experiment will, we think, throw light on the study of animal memory and cognition.

\section{REFERENCES}

Beatty, W. W., \& Shavalia, D. A. (1980). Spatial memory in rats: Time course of working memory and effects of anesthetics. Behavioral and Neural Biology, 28, 454-462.

Bloom, J. M., \& CAPAldi, E. J. (1961). The behavior of rats in relation to complex patterns of partial reinforcement. Journal of Comparative and Physiological Psychology, 54, 261-265.

Campbell, P. E., Crumbaugh, C. M., Rhodus, D. M., \& Knouse, S. B. (1971). Magnitude of partial reward and amount of training in the rat: An hypothesis of sequential effects. Journal of Comparative and Physiological Psychology, 75, 120-128.

CAPALDI, E. J. (1967). A sequential hypothesis of instrumental learning. In K. W. Spence \& J. T. Spence (Eds.), The psychology of learning and motivation (Vol. 1). New York: Academic Press.

CAPALDI, E. J. (1971). Memory and learning: A sequential viewpoint. In W. K. Honig \& P. H. R. James (Eds.), Animal memory. New York: Academic Press.

CAPALDI, E. J. (1979). Latent discrimination learning under a regular schedule of partial reinforcement. Animal Learning \& Behavior, 7 , 63-68.

CAPAldi, E. J., \& Minkoff, R. (1967). Reward schedule effects at a relatively long intertrial interval. Psychonomic Science, 9, 169-170.

CAPAldi, E. J., \& MorRIs, M. D. (1974). Reward schedule effects in extinction: Intertrial interval, memory and memory retrieval. Learning and Motivation, 5, 473-483.

CAPALDI, E. J., \& VERRY, D. R. (1981). Serial order anticipation learn- ing in rats: Memory for multiple hedonic events and their order. Animal Learning \& Behavior, 9, 441-453.

Capaldi, E. J., Verry, D. R., \& NaWrocki, T. M. (1982). Multiple hedonic memory: Memory for more than one hedonic event in rats. Animal Learning \& Behavior, 10, 351-357.

HAGGBLOOM, S. J. (1980). Reward sequence and reinforcement level as determinants of $\mathrm{S}-$ behavior in differential conditioning. Animal Learning \& Behavior, 8, 424-428.

HAGGBLOOM, S. J. (1982). Effect of N-R transitions during partial reinforcement pretraining on subsequent resistance to discrimination. Animal Learning \& Behavior, 10, 61-64.

HoNIG, W. K. (1978). Studies of working memory in the pigeon. In S. H. Hulse, H. Fowler, \& W. K. Honig (Eds.), Cognitive processes in animal behavior. Hillsdale, NJ: Erlbaum.

IsHidA, M. (1981). Persistent resistance to extinction of single alternating reinforcement after long acquisition-extinction interval. The Journal of General Psychology, 104, 191-201.

Jobe, J. B., Mellgren, R. L., Feinberg, R. A., Littlejohn, R. L., \& RIGBY, R. L. (1977). Patterning, partial reinforcement, and N-length effects at spaced trials as a function of reinstatement of retrieval cues. Learning and Motivation, 8, 77-97.

MaKI, W. S., Brokofsky, S., \& BERG, B. (1979). Spatial memory in rats: Resistance to retroactive interference. Animal learning \& $\mathrm{Be}$ havior, 7, 25-30.

Mellgren, R. L., Mays, M. Z., \& Haddad, N. F. (1983). Discrimination and generalization by rats of temporal stimuli lasting for minutes. Learning and Motivation, 14, 75-91.

OLTon, D. S. (1978). Characteristics of spatial memory. In S. H. Hulse, H. Fowler, \& W. K. Honig (Eds.), Cognitive processes in animal behavior. Hillsdale, NJ: Erlbaum.

Olton, D. S., \& SAMUElson, R. J. (1976). Remembrance of places passed: Spatial memory in rats. Journal of Experimental Psychology: Animal Behavior Processes, 2, 97-116.

SELF, R. \& GAFFAN, E. A. (1983). An analysis of serial pattern learning by rats. Animal Learning \& Behavior, 11, 10-18.

(Manuscript received 8-22-83;

revision accepted for publication 5-26-84.) 